\title{
Using a Baseline to Improve Accuracy of Waste Diversion Rate
}

\author{
Breitner S. Marczewski \\ Sr. Sustainability Engineer, General Motors, USA
}

\begin{abstract}
As part of its new sustainability goal "Zero Waste", General Motors aims to divert $90 \%$ of its total waste generation from landfills and incineration with or without energy recovery by 2025 . To achieve this goal, it is necessary to determine the correct method to track the percentage of waste diverted over time, since it will be the key performance indicator (KPI) of the program. The most accepted method to calculate this KPI is by dividing the total amount of waste diverted from landfills and/or incinerators by the total amount of waste generated in a specific time period. This methodology presents a simple snapshot of the current performance and does not fully address the long-term performance. The new proposal aims to track performance over time using similar approach as defined in the revised edition of the Greenhouse Gas Protocol. The new methodology uses a baseline to track performance and determines the diversion rate by comparing the total nondiverted material against the baseline. This method has shown accurate percentage of diversion rate and improved the company's data management since past data was used to calculate the baseline.
\end{abstract}

Keywords: Waste, Diversion Rate, Baseline, Landfill

\section{Introduction}

Waste management is the process in which residuals are managed by different methods according to its nature. According to the United States Environmental Protection Agency (EPA), there are 4 (four), categories to manage wastes as seen in the Figure 1. (U.S Environmental Protection Agency, 2021)

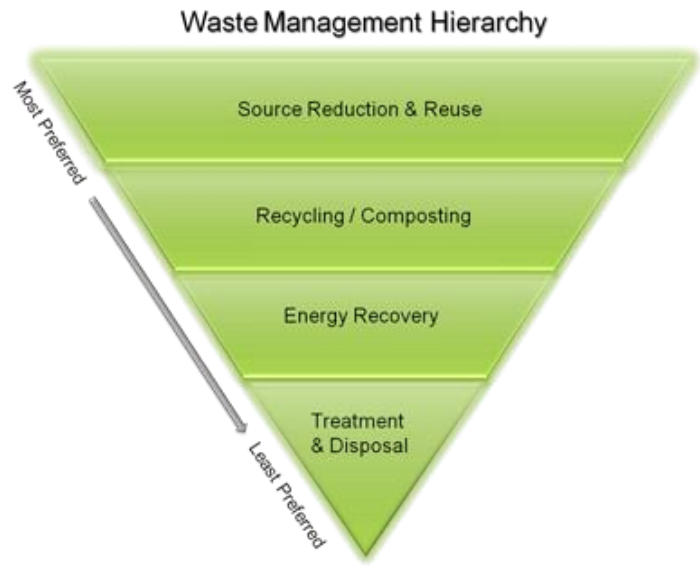

Figure 1. - EPA Waste Management Hierarchy

A waste management program comprises strategies to dispose residuals according to the waste hierarchy in Figure 1. Although debates exist regarding which method is preferred for specific waste streams (Nehrenheim, 2014), strategies in the program considers the economic, social, and environmental impacts to properly dispose wastes according to local regulations (Hoornweg \& Perinaz, 2012). Also, in terms of the goal of such program, it can be summarized as: 
"The purpose of waste management is to provide sanitary living conditions to reduce the amount of matter that enters or leaves the society and encourage the reuse of matter within the society." (Demirbas, 2011)

Therefore, to achieve this goal, it is critical to establish a methodology to measure the efficacy of the waste management program in place. One of the most common metrics is to use the diversion rate (DR) as a key performance indicator (KPI). For example, the automotive company General Motors (GM) has a public goal that aims to divert $90 \%$ of its operational solid and liquid waste from landfill and thermal processing facilities by 2025. This goal is part of the company's Zero Waste program. (General Motors Corporate Sustainability Report, 2019).

GM is not the only company to establish such sustainability goal. A research conducted by Waste Management and GreenBiz Group showed that more than $50 \%$ of the companies with $\$ 1$ billion in annual revenue have publicly committed to reduce waste-to-landfill. (Corporate Sustainability Practices: Waste \& Recycling, 2013)

Several methods have been proposed to calculate the diversion rate. The most common method is to divide the total amount diverted via recycling, reusing, composting, etc. by the total amount of waste generated and multiply the result by $100 \%$. This method, however simple, is limited and not accurate when calculating the diversion rate in the long term. The solution is to use the concept of a baseline, similarly to what the Greenhouse Gas Protocol uses for greenhouse gas emissions tracking. This, aligned with the waste hierarchy, can yield the correct diversion rate to properly assess the quality of a waste program.

Therefore, the main purpose of this study is to provide an accurate method to calculate the diversion rate of any waste management program at any given time after a baseline is established.

\section{Materials and Methodology}

A comparison between the accepted method to calculate the diversion rate was conducted using both the Total Resource Use and Efficiency (TRUE) Waste Rating System from the Green Business Certification Inc, and the Underwriters Laboratories (UL) Zero Waste to Landfill 2799 versus the new method proposed.

There are two major categories in waste management that refer to managing waste, acceptable means of diversion and non-acceptable means of diversion. The first category usually includes recycling, reusing, composting, and reductions in quantity of waste. The second usually includes landfilling and incineration (with and without energy recovery).

It is important to notice that both TRUE and the new method excludes waste-to-energy as an acceptable mean of diverting waste. Thus, any material sent to any thermal processing facility is considered non-diverted and has the same negative effect as disposing it into a landfill. However, UL2799 has a different interpretation on wasteto-energy. Its method considers this management as an acceptable mean of diversion.

The diversion rate using TRUE system is calculated using the following equation:

$$
D R_{T R U E}=\left(\frac{\text { Materials diverted from landfill, incineration }(W T E), \text { and the environment }}{\text { Total Generation }}\right) \times 100 \%
$$

Where,

$\mathrm{WTE}=$ Waste-to-Energy 
The diversion rate using UL 2799 standard is calculated using the following equation:

$$
D R_{U L}=\left\{\frac{\left(m_{\text {recycle }}+m_{\text {composting }}+m_{\text {anaerobic }}+m_{\text {reuse }}+m_{\text {reduce }}+m_{\text {TWER }}+m_{\text {biofuel }}\right)}{\left(m_{\text {dm }}-m_{\text {mandated }}\right)}\right\} \times 100 \%
$$

Where,

mrecycle $=$ mass of materials recycled

mcomposting $=$ mass of materials composted

manaerobic $=$ mass of materials sent for biodegradation in absence of oxygen

mreuse $=$ mass of materials reused

mreduce $=$ mass of materials reduced

mTWER = mass of materials sent for thermal processing with energy recovery, or also, WTE

mbiofuel $=$ mass of materials produced from biomass being sent for manufacture of or for use directly as fuel mdm $=$ mass of discarded material

mmandated $=$ materials that by local environmental regulations are disposed via thermal processing or landfill The new method to calculate the diversion is

$$
D R_{\text {new }}=\left(1-\frac{\sum m_{n d w}-\sum m_{\text {endw }}}{\sum m_{\text {baseline waste }}-\sum m_{\text {te }}}\right) \times 100 \%
$$

Where,

mndw = mass of non-diverted waste (Includes wastes managed by disposition in a landfill and thermal processing facilities)

mendw = mass of exempt non-diverted waste (Includes wastes generated in non-operational activities, such as construction, demolition, or remediation projects).

mbaseline waste $=$ mass of waste in the baseline period

mte $=$ Total mass of exempt waste

The data to complete the analysis was randomly generated and simplified to highlight the pros and cons from each method.

To test each equation, 1 (one) kilogram $(\mathrm{kg})$ in weight of non-diverted material named "Trash", $1 \mathrm{~kg}$ in weight of non-diverted material named "Used Oil", 3 (three) $\mathrm{kg}$ in weight of diverted material named "Cardboard", 
"Plastic" and "Glass" are used, and finally, $1 \mathrm{~kg}$ of non-operational waste from construction activities named "Concrete" is used. Thus, the total waste generation is 6 (six), $\mathrm{kg}$ units of residuals generated in year 1. This data is presented in table 1 below:

Table 1. - Simplified waste data to calculate the diversion rate

\begin{tabular}{lll}
\hline Waste Stream & Management Method & Mass $(\mathrm{kg})$ \\
\hline Trash & Landfill & 1 \\
\hline Used oil & Waste-to-energy & 1 \\
\hline Cardboard & Recycling & 1 \\
\hline Plastic & Recycling & 1 \\
\hline Glass & Recycling & 1 \\
\hline Concrete & Landfill (mandated) & 1 \\
\hline
\end{tabular}

A hypothetical quantity of waste streams is presented in Table 2. It considers that after a waste management program is implemented, reduction in waste streams occurs in Year 4 through Year 6.

Table 2. - Distribution of waste streams over time

\begin{tabular}{llllll} 
YEAR 1 & YEAR 2 & YEAR 3 & YEAR 4 & YEAR 5 & YEAR 6 \\
\hline Trash & Trash & Trash & & & \\
\hline Used oil & Used oil & Used oil & Trash & & \\
\hline Cardboard & Cardboard & Cardboard & Used oil & Trash & \\
\hline Plastic & Plastic & Plastic & Plastic & Used oil & Trash \\
\hline Glass & Glass & Glass & Glass & Glass & Used oil \\
\hline Concrete & Concrete & Concrete & Concrete & Concrete & Concrete \\
\hline
\end{tabular}

Table 2 shows the distribution of the waste streams over the years after a waste management program is implemented.

\section{Results}

The results are divided in 2 cases:

Case 1 - Reduction in the quantity of materials being managed by the acceptable means of diversion.

Utilizing the data from table 2, it shows that the mass diverted from landfill and incineration was $3 \mathrm{~kg}$ out $6 \mathrm{~kg}$ in Total waste generation. Applying the equation 1, the diversion rate in Year 1 is:

$$
\mathrm{DR}_{\mathrm{TRUE}}=\left(\frac{3}{6}\right) \times 100 \%=50 \%
$$

From equation 2, the UL 2799 method calculates the diversion rate in Year 1 as:

mrecycle + mTWER $=3 \mathrm{~kg}+1 \mathrm{~kg}=4 \mathrm{~kg}$ (Numerator)

mdm - mmandated $=6 \mathrm{~kg}-1 \mathrm{~kg}=5 \mathrm{~kg}$

$$
\mathrm{DR}_{\mathrm{UL}}=\left(\frac{4}{5}\right) \times 100 \%=80 \%
$$


From Year 4 to Year 6 , mreduce $=1 \mathrm{~kg}$ and needs to be added to the numerator. Also, the mdm for Year 4 to Year 6 is $5 \mathrm{~kg}, 4 \mathrm{~kg}$, and $3 \mathrm{~kg}$.

The new method uses a baseline and calculates the diversion rate based on the mass of non-diverted materials. Assuming Year 1 as the baseline, the diversion rate for that year cannot be determined using the new method since it is the reference point in time. For Year 2, the diversion rate is calculated as follows:

$$
\begin{gathered}
\sum \mathrm{m}_{\mathrm{ndw}}(\text { Trash+Used Oil+Concrete })-\sum \mathrm{m}_{\text {endw(Concrete })}=3 \mathrm{~kg}-1 \mathrm{~kg}=2 \\
\sum \mathrm{m}_{\text {baseline waste }}-\sum \mathrm{m}_{\mathrm{te}}=6 \mathrm{~kg}-1 \mathrm{~kg}=5 \mathrm{~kg} \\
\mathrm{DR}_{\text {new }}=\left(1-\frac{2}{5}\right) \times 100 \%=60 \%
\end{gathered}
$$

Utilizing the same concept, table 3 summarizes the diversion rate for each year:

Table 3. - Case 1: Diversion Rate results for each method with reduction in diverted waste

\begin{tabular}{lllllll} 
& YEAR 1 & YEAR 2 & YEAR 3 & YEAR 4 & YEAR 5 & YEAR 6 \\
\hline & Trash & Trash & Trash & & & \\
& Used oil & Used oil & Used oil & Trash & & \\
\hline & Cardboard & Cardboard & Cardboard & Used oil & Trash & \\
\hline & Plastic & Plastic & Plastic & Plastic & Used oil & Trash \\
\hline & Glass & Glass & Glass & Glass & Glass & Used oil \\
\hline $\begin{array}{l}\text { DR } \\
\text { (TRUE) }\end{array}$ & Concrete & Concrete & Concrete & Concrete & Concrete & Concrete \\
\hline $\begin{array}{l}\text { DR } \\
\text { (UL2799) }\end{array}$ & $80 \%$ & $50 \%$ & $50 \%$ & $50 \%$ & $40 \%$ & $25 \%$ \\
\hline DR (New) & $-*$ & $80 \%$ & $80 \%$ & $80 \%$ & $75 \%$ & $66.7 \%$ \\
\hline
\end{tabular}

*Baseline year 


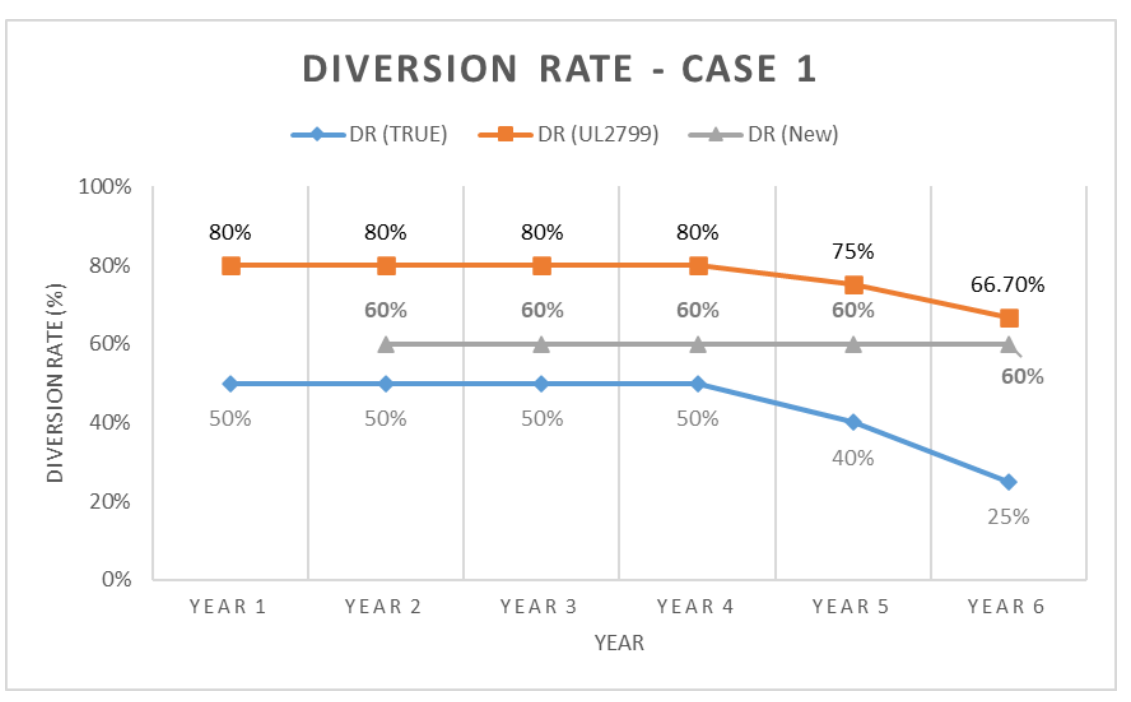

Figure 1. - Diversion rate with reduction in diverted waste

The results in table 3 show that the diversion rate, when calculated using TRUE's equation, is equal to $50 \%$ for the first 4 years, then it decreases to $40 \%$ in Year 5 and to $25 \%$ in Year 6 as the diverted waste is reduced over time. This demonstrates that this method yields a lower diversion rate even though the amount of waste is reduced over time. From a waste management standpoint, this means that the program is not being as effective as in the past years, even when reductions in the waste quantities are realized.

For the UL's method, the diversion rate is stable during the first 4 years at $80 \%$, but then it decreases in Year 5 and Year 6. This method has the same issue as the TRUE method described above. This is a serious limitation of these approaches since their scores misrepresent the overall efficiency of the waste management program in place.

The new method however, yielded the same diversion rate. This is correct because the same quantity of nondiverted material did not change over time compared to the baseline.

Case 2 - Reduction in the quantity of materials being managed by the non-acceptable means of diversion.

A second evaluation is performed by reducing the amount of non-diverted materials from Year 1 to Year 6. The quantities and the new diversion rates are shown in table 4 below:

Table 4. - Case 2: Diversion Rate results with reduction in non-diverted waste

\begin{tabular}{lllllll} 
& YEAR 1 & YEAR 2 & YEAR 3 & YEAR 4 & YEAR 5 & YEAR 6 \\
\hline Trash & Trash & Trash & & & \\
\hline & Used oil & Used oil & Used oil & Trash & & \\
\hline & Cardboard & Cardboard & Cardboard & Used oil & Trash & \\
\hline & Plastic & Plastic & Plastic & Plastic & Plastic & Plastic \\
\hline & Glass & Glass & Glass & Glass & Glass & Glass \\
\hline $\begin{array}{l}\text { Concrete } \\
\text { (TRUE) }\end{array}$ & Concrete & Concrete & Concrete & Concrete & Concrete \\
\hline $\begin{array}{l}\text { DR } \\
\text { (UL2799) }\end{array}$ & $80 \%$ & $50 \%$ & $50 \%$ & $50 \%$ & $60 \%$ & $75 \%$ \\
\hline & $80 \%$ & $80 \%$ & $80 \%$ & $75 \%$ & $100 \%$
\end{tabular}




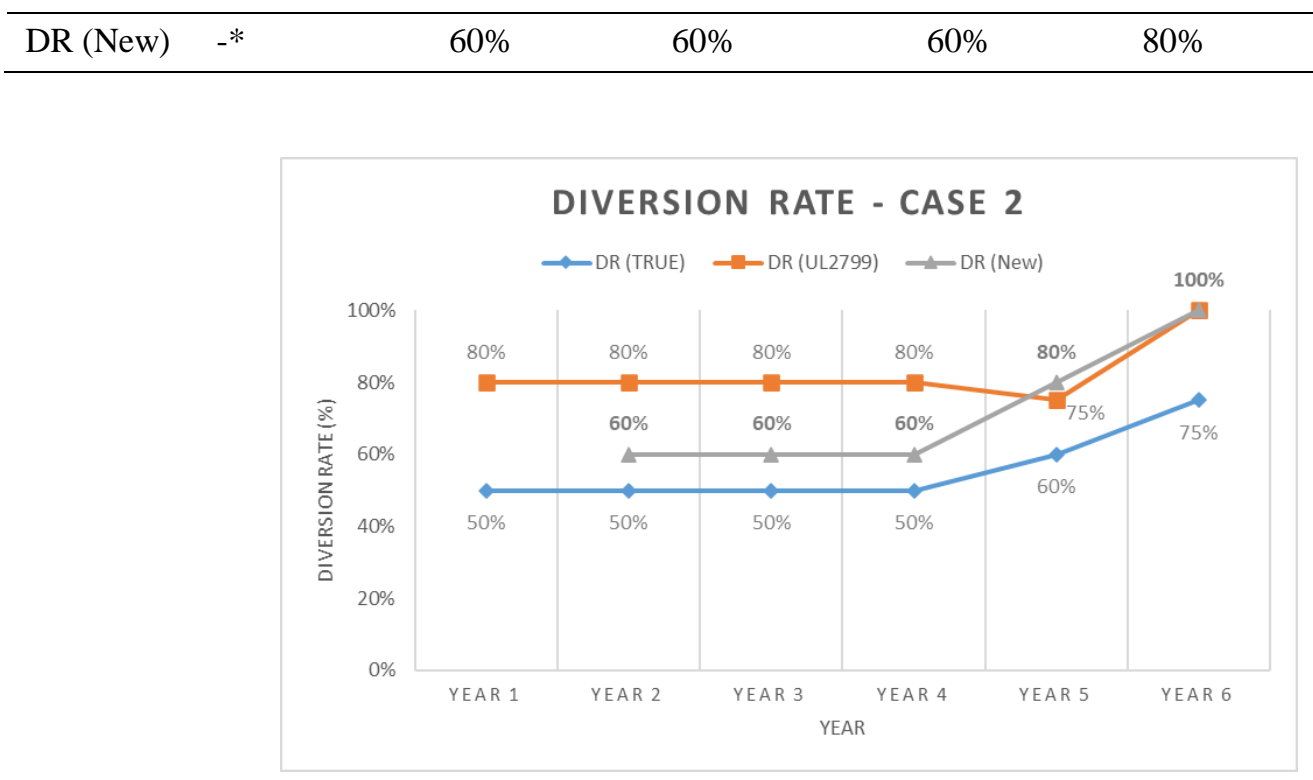

Figure 2 - Diversion rate with reduction in non-diverted waste

The results in table 4 shows that the diversion rate, when calculated using TRUE's equation, is equal to $50 \%$ for the first 4 years, then it increases to $60 \%$ in Year 5 and to $75 \%$ in Year 6 as the diverted waste is reduced over time. This demonstrates that this method yields a higher diversion rate when the amount of non-diverted waste is reduced over time.

For the UL's method, the diversion rate is stable during the first 4 years at $80 \%$, but then it decreases in Year 5 to $75 \%$ and then increases to $100 \%$ in Year 6. This method is inaccurate since the diversion rate yields a lower percentage in the subsequent year after the reduction in non-diverted waste.

The new method however, yielded the correct diversion rate over time. The percentage value increases as the non-diverted amount is reduced in any given year compared to the baseline.

\section{Conclusion}

Based on the results of the diversion rate, both TRUE and UL methods indicate lower diversion rate when diverted materials are reduced over the years. From a waste management standpoint, it misleads the assessment that the current program in place is not being effective. Utilizing the novel method, however, proves to indicate accurate performance over time, even when changes exist in both weights of non-diverted and diverted material.

Establishing a baseline as a reference is the key to correct the misleading diversion rate percentages. If the UL or TRUE methods are to be used, their results should be interpreted more as a snapshot of the current efficiency of a waste management program with reservations. If the program is new, and no sustainability or waste reduction efforts had been in place before, then they could provide a reasonable assessment of the status of the waste profile. If not, it is best to determine a baseline for comparison and follow the calculations proposed.

The new method has the advantage to fix the issues with the quantities of waste being reduced over time. The disadvantage of the new method is the lack of credit for reductions in materials managed by acceptable means of diversion. This means that, a waste management program will not receive any benefits in reducing the quantity of materials already managed by the acceptable means of diversion. The new method, as is, will show a better diversion rate only when waste streams managed by non-acceptable means of diversion are reduced (pushed upwards in the waste hierarchy) over time. Therefore, the model could be improved if it could incorporate methods to promote reductions of waste generation, regardless where it stands in the waste hierarchy. 
Nevertheless, the new method yields the correct diversion and could assess the efficacy of any waste management program when the goal is to divert waste from landfills alone or including thermal processing facilities.

\section{Acknowledgement}

This work was only possible due to the dedication and great support from my manager: Geraldine Barnuevo. Her relentless pursuit to make the world a better place inspires all professionals at General Motors. My mentor Rusty Helm, who always provides me with great career insights and feedback. Glenn Perham, Megan Karie, Kaitlyn Miller and Sarah Tea, my colleagues at General Motors, have always provided outstanding support and feedback in the conception of our new Zero Waste program. I am also grateful for General Motors leadership for understanding the pressure to advance sustainability in the automotive industry.

\section{References}

Corporate Sustainability Practices: Waste \& Recycling (2013). Retrieved from

http://info.greenbiz.com/rs/greenbizgroup/images/Corporate\%20Sustainability\%20Practices\%20\%20Waste\%20Reduction\%20Report.pdf?mkt tok=3RkMMJWWfF9wsRojuajOZKXonjHpfsX86uwvUKeylMI \%2F0ER3fOvrPUfGjI4FSsdkI\%2BSLDwEYGJlv6SgFSLHEMa5qw7gMXRQ\%3D

Demirbas, A. (2011). Waste management, waste resource facilities and waste conversion processes. (2011). Energy Conversion and Management, 52(2), 1280-1287. Retrieve from https://doi.org/10.1016/j.enconman.2010.09.025.

E. Nehrenheim, (2014). Waste Management: Introduction, Reference Module in Earth Systems and Environmental Sciences, Elsevier. https://doi.org/10.1016/B978-0-12-409548-9.09166-1

General Motors Corporate Sustainability Report (2019). Retrieved from

https://www.gmsustainability.com/material-topics/designing-for-the-environment.html

Hoornweg, D., \& Bhada-Tata, P. (2012). What a Waste: A Global Review of Solid Waste Management. Urban development series; knowledge papers no. 15. World Bank, Washington, DC. (C) World Bank. https://openknowledge.worldbank.org/handle/10986/17388

TRUE Zero Waste System (2020, June). Retrieved from

https://true.gbci.org/sites/default/files/resources/TRUE_RatingSystemGuide_r1.pdf

Underwriters Laboratories 2799 Environmental Claim Validation Procedure for Zero Waste to Landfill (2017, March). Retrieved from

https://www.shopulstandards.com/ProductDetail.aspx?UniqueKey=32526

U.S Environmental Protection Agency (EPA), Waste Management Hierarchy and Homeland Security Incidents. Retrieved from

https://www.epa.gov/homeland-security-waste/waste-management-hierarchy-and-homeland-security-incidents 